\title{
MyD88 provides a protective role in long-term radiation-induced lung injury
}

\author{
Willie J. Brickey ${ }^{1}$, Isabel P. Neuringer ${ }^{2}$, William Walton ${ }^{3}$, Xiaoyang Hua $^{2}$, Ellis Y. Wang ${ }^{3}$, \\ Sushmita Jha ${ }^{3}$, Gregory D. Sempowski ${ }^{4}$, Xuebin Yang $^{5}$, Suzanne L. Kirby ${ }^{3,5}$, Stephen L. \\ Tilley ${ }^{2}$, and Jenny P-Y. Ting ${ }^{1,3}$ \\ ${ }^{1}$ Department of Microbiology/Immunology, University of North Carolina at Chapel Hill \\ 2Department of Pulmonary and Critical Care Medicine, University of North Carolina at Chapel Hill \\ ${ }^{3}$ Lineberger Comprehensive Cancer Center, University of North Carolina at Chapel Hill \\ ${ }^{4}$ Duke Human Vaccine Institute, Duke University, North Carolina \\ ${ }^{5}$ Department of Pathology and Laboratory Medicine, University of North Carolina at Chapel Hill, \\ North Carolina, USA
}

\begin{abstract}
Purpose-The role of innate immune regulators is investigated in injury sustained from irradiation as in the clinic for cancer treatment or from a nuclear incident. The protective benefits of flagellin signaling through Toll-like receptors (TLR) in an irradiation setting warrant study of a key intracellular adaptor of TLR signaling, namely Myeloid differentiation primary response factor 88 (MyD88). The role of MyD88 in regulating innate immunity and Nuclear factor kappa-B (NF-kB)-activated responses targets this critical factor for influencing injury and recovery as well as maintaining immune homeostasis.
\end{abstract}

Materials and methods-To examine the role of MyD88, we examined immune cells and factors during acute pneumonitic and fibrotic phases in Myd88-deficient animals receiving thoracic gamma $(\gamma)$-irradiation.

Results-We found that MyD88 supports survival from radiation-induced injury through the regulation of inflammatory factors that aid in recovery from irradiation. The absence of MyD88 resulted in unresolved pulmonary infiltrate and enhanced collagen deposition plus elevated type 2 helper T cell (Th2) cytokines in long-term survivors of irradiation.

Conclusions-These results based only on a gene deletion model suggest that alterations of MyD88-dependent inflammatory processes impact chronic lung injury. Therefore, MyD88 may contribute to attenuating long-term radiation-induced lung injury and protecting against fibrosis.

\section{Keywords}

Radiation; inflammation; innate immunity; MyD88; lung injury

\section{(C) 2012 Informa UK, Ltd.}

Correspondence: Dr Jenny P-Y. Ting, 450 West Drive, Campus Box \#7295, 22-004 Lineberger Comprehensive Cancer Center, University of North Carolina at Chapel HillChapel Hill, NC 27599, USA. Tel: + 1919966 5538. Fax: + 19199668212. jenny_ting@med.unc.edu.

Declaration of interest: The authors report no conflicts of interest. The authors alone are responsible for the content and writing of the paper. 


\section{Introduction}

The lung is a highly radiosensitive organ which represents a prime tissue targeted for damage due to irradiation (Chen et al. 2002). Direct injury of the endothelial and epithelial tissues in the lung is a crucial clinical outcome of irradiation, leading to acute pneumonitis and delayed fibrosis. Radiation pneumonitis is an early response to radiation injury, resulting from immune and lung parenchymal cell interactions, accompanied by proinflammatory cytokines and chemokines (Chen et al. 2002, Ao et al. 2009), while fibrosis is manifested with late lung injury and/or unresolved inflammatory responses. Both cells of the adaptive immune system, such as thymic-derived lymphocytes (T cells), and of the innate immune system, such as inflammatory granulocytes or macrophages, have been implicated in radiation-induced injury (Roberts et al. 1993, Nakayama et al. 1996, Miura et al. 2000). A role for immune regulation in radiation pneumonitis has been demonstrated by: (i) The correlation of enhanced proinflammatory cytokines, specifically of Interleukins one alpha and six (IL-1 $a$ and IL-6), with the incidence of radiation pneumonitis (Chen et al. 2002); (ii) the association of prior thymectomy and a lowered incidence of radiation pneumonitis (McBride and Vegesna 2000); (iii) an increased T cell infiltrate in patients who later develop radiation pneumonitis compared to those who did not (Roberts et al. 1993, Nakayama et al. 1996); and (iv) an association of decreased Tumor necrosis factor alpha (TNFa) caused by treatment with pentoxifylline with ameliorated radiation pneumonitis (Rube et al. 2002).

Radiation injury is sustained as a consequence of radiotherapy for cancer as well as by incidental exposure in the case of nuclear accidents or even bioterrorist acts. In either case, protective measures are important for preventing lethality and for providing amelioration of off-target effects to non-diseased cells or tissues. Activation of Toll-like receptor (TLR) signaling, namely toll-like receptor 5 (TLR5) through the exposure to flagellin, has been reported to be very effective in protecting cells, mice and non-human primates from radiation-induced lethality (Burdelya et al. 2008, Vijay-Kumar et al. 2008). It is becoming increasingly important to understand the protective roles supported by TLR signaling in order to develop immunotherapies that synergize with radio- and chemotherapeutic approaches for the treatment of cancer patients (Roses et al. 2008).

TLR signaling in mammals has rapidly emerged as a major route by which the immune system responds to microbial pathogens, pharmacologics, synthetic compounds as well as non-infectious stress insults through activation and regulation of cell proliferation and differentiation programs (Medzhitov and Janeway 2000, Akira and Takeda 2004, Beutler 2004, Jiang et al. 2006, McGettrick and O' Neill 2007). TLR activation requires a host of intracellular adaptor proteins proximal to the TLR, with the most prominent being Myeloid differentiation primary response factor 88 (MyD88) (Cao et al. 1996, Hacker et al. 2000, Wang et al. 2001). With the exception of a subset of toll-like receptors 4 and 3 (TLR4 and TLR3) stimulations in vivo, all TLR studied to date display MyD88-dependent signaling; thus an analysis of mice lacking the MyD88 molecule provides a targeted assessment of the role of TLR signaling in a specific biologic outcome. Although previous human (Liebermann and Hoffman 2003, Li 2004) and animal studies with Myd88-null mice have demonstrated roles of this molecule in atherosclerosis (Bjorkbacka et al. 2004), cancer adjuvant therapy (Akazawa et al. 2004), onset of autoimmune disorder, and allotransplant rejection (Goldstein et al. 2003), the in vivo role of MyD88 in radiation-induced pulmonary injury has not been studied.

Supported by the evidence for roles of adaptive and innate immune responses in radiationinduced pneumonitis, the focus of this report is to determine the role of MyD88, a specific key regulator of immunity, in radiation-induced injury. This molecule controls innate 
immunity and proinflammatory responses through its mediation of intracellular signaling (Kenny and O' Neill 2008). It is a cytosolic adaptor molecule that acts as an intermediary between activated TLR, interleukin 1 or 18 receptors (IL-1R/IL-18R) and other signaling molecules that are proximal to the membrane. MyD88 contains two prominent domains, the T oll-like receptor/I nterleukin 1 Receptor (TIR) domain and a death domain (Xu et al. 2000). The TIR domain consists of an alpha-beta fold and forms a complex with its associative membrane receptors (TLR, IL-1R and IL-18R) when the latter become activated by their respective agonist or ligand. Upon activation, the death domain of MyD88 interacts with the death domain of IL-1 receptor-associated kinase-1 (IRAK1). This results in the recruitment of a membrane proximal complex consisting of TNF receptor associated factor 6 (TRAF6), IL-1 receptor-associated kinases-1, 2, or 4 (IRAK1/2/4) and transforming growth factor- $\beta$-activated kinase-1 (TAK-1). Further downstream mediators include nuclear factor$\kappa$ B-inducing kinase (NIK), Inhibitor of nuclear factor- $\kappa$ B kinase (IKK), Mitogen-activated protein kinase kinase 1 (MEKK1), and c-jun N-terminal kinase (JNK) that lead to the endpoint regulators of gene activation, namely nuclear factor-kappaB (NF- $\mathrm{kB}$ ) and Mitogen-activated protein kinase (MAPK)-induced activities. NF- $\mathrm{BB}$ and MAPK regulate downstream genes that include an array of cytokines, chemokines, growth factors and cell surface molecules which can significantly influence innate and adaptive immunity.

The pivotal role MyD88 plays in transmitting intracellular signals from activated innate immune receptors to the transcriptional regulator NF- $\mathrm{kB}$ suggests that elimination of this gene will significantly alter immune responses to danger, stress or pathogenic stimuli. Although architectural and physiologic changes in lungs have long been recognized as adverse events due to radiation exposure, immune mechanisms regulating and/or protecting against injury are less understood (Abid et al. 2001, Hill 2005, Graves et al. 2010). Here, the pulmonary consequences of loss of MyD88 to radiation- induced stress have been tested by treating $M y d 88$-null animals with a single high dose of gamma $(\gamma)$-irradiation to the thorax. To discern contributions of MyD88 to radiation-induced pulmonary injury and/or repair during acute and late lung injury phases, the following outcomes were monitored: Survival, pathology and inflammatory factors (Williams et al. 2010). Mice lacking MyD88 were more susceptible to death due to thoracic exposure to irradiation and displayed fibrosis hallmarks such as increased collagen deposition and dysregulated cytokines. Based solely on examination of gene-deficient mice, these initial results suggest that not only is MyD88 important for homeostasis (Nagai et al. 2006, McGettrick and O' Neill 2007), but it also appears to confer a protective role against radiation-induced lung injury. This initial characterization should pave the way for further study, possibly using cell specific targeting of MyD88 function as well as biochemical approaches, to identify MyD88 as a critical molecule for radiation countermeasures.

\section{Materials and methods}

\section{Animals}

Male mice, aged 8-12 weeks old, were used in these studies. C57BL/6 mice (or wild type, WT) originally from Jackson Laboratory (Bar Harbor, ME, USA) and bred at The University of North Carolina at Chapel Hill (UNC-CH), NC, USA along with congenic Myd $88^{-1-}$ mice (originally from Dr Shizou Akira, Osaka University, Osaka, Japan) backcrossed to C57BL/6 mice for at least nine generations from Dr Donald Cook, National Institute of Environmental Health Sciences (NIEHS), Research Triangle Park, NC, USA were examined. All animals were housed in an Association for the Assessment and Accreditation of Laboratory Animal Care accredited pathogen-free facility. All animals were treated humanely according to National Institutes of Health (NIH) guidelines and procedures approved by UNC-CH's Institute of Animal Care and Use Committee. 


\section{Radiation-induced injury model}

Mice received a single dose of thoracic-targeted irradiation ( 14 gray [Gy]) using a collimator (Model 335) with a ${ }^{137}$ Cesium $\gamma$-ray source (Model Mark I-68; J.L. Shepherd \& Associates, San Fernando, CA, USA) at a $1.7 \mathrm{~Gy} / \mathrm{min}$ dose rate. Prior to irradiation, mice were anesthetized with tribromoethanol (Sigma-Aldrich Corp., St Louis, MO, USA) and then loosely immobilized over a $2.9 \mathrm{~cm}^{2}$ opening in a lead block (manufactured by UNC$\mathrm{CH}$ Radiology). The treated mice as well as untreated age-matched controls were housed in cages in pathogen-free rooms and given standard laboratory chow and water ad libitum. Routine serological testing of sentinel animals housed alongside the irradiated animals revealed the absence of mites, pinworms, Mycoplasma pulmonis, and a host of viruses, including epizootic diarrhea of infant mice (EDIM), Theiler's murine encephalomyelitis virus (TMEV GDVII strain), Mouse Hepatitis Virus (MHV), Murine minute virus (MMV), Murine parvovirus (MPV) and non-structural protein-1 (NS-1) antigen, Pneumonia Virus of Mice (PVM), and Sendai virus. Experimental and control mice were sacrificed at designated times up to 27 weeks post irradiation (wpi) for analyses.

\section{Peripheral blood assessment}

Hematological tests to determine abundance of lymphocytes, granulocytes and monocytes in the white blood cell (WBC) fraction on ethylenediaminetetraacetic acid (EDTA)-whole blood collected by retro-orbital or submandibular bleeding were performed using an Animal Blood Counter (Heska, Loveland, Colorado, USA) in the Animal Clinical Chemistry Facility at UNC-CH.

\section{Lung histopathology}

Harvested lung tissues were inflated with $10 \%$ formalin (Sigma-Aldrich), paraffinembedded and sectioned (5 micron) at the Animal Histopathology Core Facility at UNCCH. Prepared sections of intact lobe were stained with hematoxylin and eosin (H\&E), Masson's trichrome (MT) (Sigma-Aldrich) or Picosirius Red (SR) (Polysciences Inc, Warrington, PA, USA) and viewed by light microscopy (ScanScope microscope, Aperio Technologies, Vista, CA, USA). Several pathologic features plus degree of cellularity were assessed by reviewers unaware of the identity of each sample. Each interstitial focal infiltrate found in the lung section was graded according to the following scale: 0 , no apparent abnormality; 1, cellularity encompassing an area $<25$ alveolar spaces; 2 , an area between 25-50 alveolar spaces; 3 , an area between 50-100 alveolar spaces; and 4, an area $\geq$ 100 alveolar spaces. The corresponding grades for all the foci per lung sample were combined to give a cellularity score. Collagen content was determined indirectly by assessing hydroxyproline levels in the right lung (Smith et al. 1994, Keane et al. 1999) or by SR staining (Junqueira et al. 1979), where images of seven randomly chosen fields localized to the subpleural regions were captured using a polarizing filter on a BX61 microscope (Olympus, Center Valley, PA, USA), with averaged pixels of polarized light determined by ImageJ analysis (available at NIH via http://rsbweb.nih.gov/ij/). Immunohistochemistry was performed on deparaffinized and re-hydrated lung tissue sections to examine $\mathrm{T}$ cells (hamster anti-mouse T cell receptor beta or TCR $\beta$ antibody conjugated with phycoerythrin, 1:200 dilution, H57-597 clone; Beckman Coulter Incorporated, Miami, FL, USA), and myeloid cells (rat anti-mouse Integrin alpha $\mathrm{M}$ or cell differentiation marker $11 \mathrm{~b}, \mathrm{CD} 11 \mathrm{~b}$, antibody conjugated with allophycocyanin, 1:200 dilution, M1/70 clone; eBioscience, San Diego, CA, USA) as described (Arnett et al. 2002, Jha et al. 2010). Sections were mounted in VECTASHIELD ${ }^{\circledR}$ with 4' ${ }^{\prime}$, 6-diamidino-2-phenylindole (DAPI) (Vector Laboratories, Burlingame, CA, USA) and viewed using an Olympus BX-40 microscope fitted with DP72 camera. Images were captured using cellSens ${ }^{\circledR}$ image acquisition software (Olympus) and immunopositive cells with an observable DAPI-stained nucleus were counted in 5-7 randomly chosen fields per lung section. 


\section{Ribonucleic acid (RNA) profiling}

Total RNA was generated from lung homogenates using Trizol (Invitrogen Corporation, Carlsbad, CA, USA), and converted into complementary deoxyribonucleic acid (DNA) using random hexamers and Moloney Murine Leukemia Virus-Reverse Transcriptase (MMLV-RT) (Invitrogen), according to manufacturer's instructions. Then, polymerase chain reaction (PCR) amplification was performed using gene specific primers with probes or with cyanine dye (i.e., SYBR green) incorporation using a 7900T Fast Real-Time PCR System (Applied Biosystems Incorporated or Life Technologies Corporation, Carlsbad, CA, USA). Primer sequences included: ribosomal RNA (i.e., 18 Svedberg unit, 18S rRNA) (tetrachlorofluorescin or TET-CAAATTACCCACTCCCGACCCG- black hole quencher or BH, 5' -GCTGCTGGCACCAGA CTT-3' ' 5'-CGGCTACCACATCCAAGG-3'); Transforming growth factor beta $(T g f b)$ (6-carboxyfluorescein or FAMACCTTGGTAACCGGCTGCTGACC -tetramethylrhodamine quencher or TAMRA, 5' -GGAACTCTACCAGAAA TATAGC-3' ${ }^{\prime}, 5^{\prime}$-CACTCAGGCGTATCAGTG-3' ${ }^{\prime}$ ); Forkhead box P3 (FoxP3) (FAM-ATCCTACCCACTGCTGGCAAATGGAGTC-TAMRA, 5'-CCCAGGAAAGACAGCAACCTT-3' ${ }^{\prime}$ 5' -TTCTCACAACCA GGCCACTTG-3'); Interleukin 1beta $(I I 1 b)\left(5^{\prime}\right.$-CTCATTGT GGCTGTGGAGAAG-3' ${ }^{\prime} 5^{\prime}$ ACCAGCAGGTTATCATCATCAT-3'); Interleukin 6 (Il6) (5' TACCATAGCTACCTGGAGTACAT-3 ' 5 ' '-CTGTGACTCCAGCTTATCTGTTA-3 '). Commercial primer/probe sets (Life Technologies Corporation) for Chemokine (C-C motif) ligand 2 or Monocyte chemoattractant protein 1 (Ccl2/Mcp1; Mm00441242_m1); Chemokine (C-X-C motif) ligand 1 or platelet-derived growth factor-inducible $\mathrm{KC}$ protein (Cxcl1/KC; Mm00433859_m1); Endothelin 1 (Edn1; Mm00438656_m1); Hyaluronan synthase 2 or 3 (Has2; Mm00515089_m1 or Has3; Mm00515091_m1); Intercellular adhesion molecule (Icam1; Mm00516023_m1); Matrix metalloproteinase 2 (Mmp2; Mm00439506_m1); Myeloperoxidase (Mpo; Mm01298424_m1); and extracellular superoxide dismutase 3 (Sod3; Mm00448831_m1) were utilized. Genespecific transcripts were first normalized to $18 \mathrm{~S}$ rRNA transcripts for each sample and then ratios of normalized RNA in treated samples to untreated controls were generated. Fold change in expression from untreated samples are reported.

\section{Protein profiling}

Serum was prepared from blood taken by cardiac puncture and profiled using the Mouse Cytokine 20-Plex Panel (Invitrogen). Mean protein \pm standard error of the mean (SEM) (pg/ $\mathrm{ml})$ are reported with each group consisting of at least three biological replicates $(n=3-5)$.

\section{Lung physiology assessments}

Physiologic measures of lung function in mice surviving for more than 18 wpi were assessed as described (Irvin and Bates 2003, Lovgren et al. 2006, Glaab et al. 2007). Briefly, mice were anesthetized, tracheostomized, and mechanically ventilated using a computercontrolled small animal ventilator or FlexiVent (SCIREQ Incorporated, Montreal, Quebec, Canada). Pressure-volume curves were used to determine static compliance (Cst) and oscillatory mechanics were used to determine tissue elastance $(\mathrm{H})$ of the lungs of treated and control mice.

\section{Statistics}

Student's $t$ - test and Mann-Whitney test were used to contrast results for WT and $M y d 88^{-1-}$ at similar conditions as indicated by dotted lines, while solid lines represent analysis by one way analysis of variance (ANOVA) for a single genotype over time. Differences with $P$ values $<0.05$ were considered significant. Kaplan-Meier survival curves were generated using Prism (GraphPad Software Inc., La Jolla, CA, USA). 


\section{Results}

\section{$M y d 88^{-/-}$mice are susceptible to death due to radiation-induced lung injury}

To begin to define the in vivo role of MyD88 in radiationinduced injury, we followed a cohort of irradiated $\mathrm{Myd} 88^{-/-}$mice over time to determine if loss of MyD88 affected organism survival to thoracic irradiation. Targeting radiation to the thorax while also shielding the body necessitated an experimental set-up whereby mice were anesthetized (via intraperitoneal injection of tribromoethanol) immediately prior to immobilization over a 2.9 $\mathrm{cm}^{2}$ opening in a lead block. Anesthetized mice became alert after radiation exposure in the same time course irrespective of genotype, suggesting no adversity correlating with genetic differences. Overall, we inferred a protective role for MyD88 as the absence of MyD88 sensitized mice to die sooner (Figure 1A). More than half of the irradiated $\mathrm{Myd} 88^{-1-}$ mice were dead by 28 weeks post irradiation (wpi), and $80 \%$ of these mice were dead by the end of the experiment ( $33 \mathrm{wpi}$ ), while the majority of treated wild type (WT) animals survived the entire course of the experiment.

Peripheral blood cell composition in WT and $\mathrm{Myd} 88^{-/-}$mice prior to and after irradiation was examined. Although not significantly divergent from WT, a trend of diminished cell numbers in $\mathrm{Myd} 88^{-1-}$ mice at baseline was seen (Figure 1B). Dynamic changes in peripheral blood cell types were followed for six weeks during the acute response. Similar kinetic

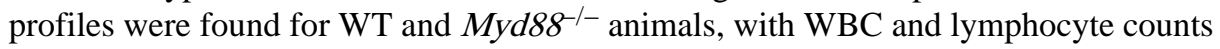
dropping precipitously by three days and then recovering to or above baseline levels by 6 wpi (Figure 1C). Although granulocytes and monocytes comprised a smaller portion of the peripheral WBC in absolute numbers, these cell types were relatively static near baseline levels throughout six weeks following irradiation for WT and $M y d 88^{-/-}$mice, with minor differences in monocytes at 1.4 weeks and granulocytes prior to radiation in $M y d 88^{-/-}$ mice.

\section{Increased cellularity is evident in the lungs of treated $M y d 88^{-1-}$ mice during acute injury}

To examine the injury phenotype in the absence of MyD88, we investigated pulmonary pathology associated with thoracic irradiation. H\&E stained lung tissues from treated mice during six weeks after irradiation were examined for cellular infiltrate (Figure 2A). An interstitial infiltrate score estimating the number of foci as well as the area of alveolar spaces spanned by infiltrate was assigned by reviewers unaware of the identity of genotype or time after treatment. These semi-quantitative observations indicated increased cellularity in lungs of $M y d 88^{-/-}$mice by 4-6 wpi, whereas WT tissues appeared to have low levels of infiltrate similar to the basal state (Figure 2B).

To begin to address the nature of the cellular infiltrate, immune cell populations in the lung were investigated by cell-specific antibody staining of fixed lung sections. T and myeloid cells were detected by TCR $\beta$ and CD11b immunohistochemistry, respectively (Figure 2C). Examination of these stained sections revealed elevated numbers of TCR $\beta^{+}$and CD11 $\mathrm{b}^{+}$ cells in tissues after irradiation, but with no differences in numbers of immunopositive cells between WT and $M y d 88^{-/-}$sections (Figure 2D). (Neutrophils, macrophage and other cell types or activation states were not specifically distinguished.)

\section{Focal damage is evident in long-term surviving radiation-treated $M y d 88^{-1-}$ mice}

We anticipated that the events occurring during the acute phase of inflammation would influence the course of repair and long-term pathology (Rubin et al. 1995). Therefore we examined long term irradiated $M y d 88^{-/-}$and corresponding WT survivors up to 27 weeks after treatment. Upon histologic examination of fixed lung tissues (Figure 3A), we found more sites of damage encompassing between 25 and 250 alveolar spaces in long-term 


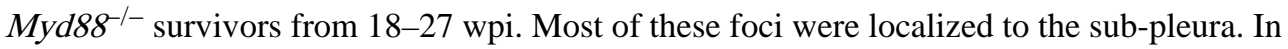
contrast, foci in irradiated WT survivors were sparse, appearing between 24 and 27 wpi. The wet weights of the right lobes of irradiated WT and $\mathrm{Myd} 88^{-1-}$ mice progressively increased from 13-27 wpi, with $M y d 88^{-/-}$lungs trending toward greater mass at each respective time (Figure 3B). Similarly, a dynamic change in body weight, with a loss at $18 \mathrm{wpi}$, followed by a rebound increase by 24-27 wpi was evident for WT and $M y d 88^{-1-}$ mice with radiation treatment (Figure 3C).

The link between pulmonary pathology and altered airway function was determined by assessing respiratory mechanics of unirradiated and treated WT and $M y d 88^{-1-}$ mice. Static lung compliance (Cst), defined as ratio of change in lung volume over change in pressure under static conditions of zero flow, and tissue elastance $(\mathrm{H})$, an indicator of the degree of elasticity or rigidity of the lung with forced oscillatory flow, were measured as described (Lovgren et al. 2006, Glaab et al. 2007). For WT, compliance dropped over the course of examination from 18-27 wpi (Figure 3D). For $M y d 88^{-/-}$mice, compliance decreased significantly from 18-24 wpi over unirradiated controls. Examination of the tissue elastance

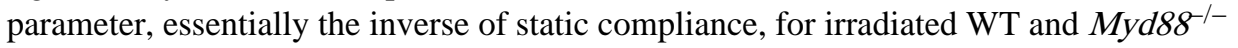
mice, revealed increasing values, signifying progressively more rigid lungs in response to forced inflations. Overall, biological parameters observed during the late lung injury phase for irradiated WT and $M y d 88^{-1-}$ mice were very similar, except for the increased incidence of parenchymal damage localized to the subpleura in lungs of irradiated $M y d 88^{-1-}$ mice.

\section{Densely-packed collagen-filled nodules are localized to sub-pleura of lungs of irradiated Myd88-/- mice}

As radiation-induced fibrosis evident in C57BL/6 mice (and related strains) appears to be heterogeneous (Sharplin and Franko 1989, Dileto and Travis 1996, Chiang et al. 2005, Jackson et al. 2010, Williams et al. 2010), multiple semi-quantitative approaches were utilized to examine collagen deposition. These indirect assessments included histological staining with Masson's Trichrome (MT) or Picosirius Red (SR) and biochemical analysis of hydroxyproline content, a surrogate marker of collagen. MT staining revealed that the nodules observed at low power in H\&E stained whole lung lobes in treated $M y d 88^{-/-}$mice at 24-27 wpi were densely composed of collagen, with similar foci being noticeably diminished in irradiated WT lung tissue (Figure 4A). SR staining showed collagen deposition in the sub-pleural foci of lungs of irradiated $M y d 88^{-/-}$mice at 24-27 wpi (Figure 4B), in contrast to minimal sub-pleural localized collagen in irradiated WT mice between 24 and 27 wpi. Consistent with these histological findings, albeit more limited in scope, biochemical assessment of hydroxyproline content showed that hydroxyproline was considerably higher in the lungs of long term $\mathrm{Myd} 88^{-1-}$ survivors at 24 wpi than in untreated $\mathrm{Myd} 88^{-1-}$ or WT long-term survivors at 24 wpi (data not shown). Semiquantitative evaluation of SR-stained sections using polarized light microscopy indicated elevated levels of collagen fiber at $27 \mathrm{wpi}$ in $\mathrm{Myd} \mathrm{s}^{-/-}$mice in comparison to WT mice at that time (Figure 4C). Taken together, these results revealed the presence of elevated collagen associated with a fibrotic phenotype in long term $\mathrm{Myd} 88^{-/-}$survivors of irradiation.

Radiation-induced lung injury in $M y d 88^{-/-}$mice is associated with altered inflammatory, chemotactic and fibrotic transcriptome responses. We further investigated the immune potential of the cellular infiltrate by profiling the expression of pro-inflammatory and profibrogenic factors. Lung tissue from WT and $M y d 88^{-/-}$strains was harvested from mice during the first six weeks after irradiation and total RNA was isolated. Gene-specific PCR reagents were used to quantify changes in transcripts in lung tissue. 
The most outstanding changes in expression of inflammatory factors in WT animals due to radiation stress included inductions of RNA expressing proinflammatory Interleukin-6 (Il6; 10- to 45-fold), Myeloperoxidase ( $\mathrm{Mpo}$; 10 - to 25 -fold), Monocyte chemoattractant protein 1 (Ccl2/Mcp1; 7-fold), and Chemokine (C-X-C motif) ligand 1 (CXcl1/KC; > 10-fold) (Figure 5). Increases were most dramatic at 4 wpi, and generally subsided by 6 wpi. In contrast, the induced expression of II6, Mpo, Ccl2/Mcpl, and Cxcl1/KC was ameliorated in irradiated $M y d 88^{-/-}$animals. Likewise, transcripts for extracellular Superoxide dismutase encoded by $S o d 3$, Transforming growth factor-beta $(T g f b)$ and Intercellular adhesion molecule 1 (Icam1) were modestly induced in irradiated WT mice (2- to 3-fold between 10 days and 4 wpi), while these transcripts were only transiently induced by 10 days or not at all in irradiated $M y d 88^{-1-}$ mice. In contrast, the expression of Interleukin-1- beta (IIIb) and Hyaluronan synthetases 2 and 3 (Has2, Has3) transcripts were elevated (2- to 12-fold, primarily by 7-10 days after irradiation) in $\mathrm{Myd} 88^{-1-}$ mice, but not in treated WT mice. Lastly, transcripts encoding a T cell marker (i.e., Forkhead Box P3 or FoxP3) were similarly regulated over six weeks between WT and $\mathrm{Myd} 88^{-/-}$samples, consistent with the similar TCR $\beta$ immunostaining pattern (Figure 2). Although still a possibility, this observation

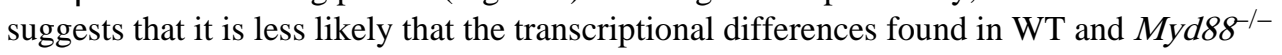
lungs after radiation are due to alterations in cell recruitment to the lung.

\section{Pro-fibrogenic proteins are abundant in long-term $\mathrm{Myd} 8^{-/-}$survivors of radiation- induced lung injury}

Lastly, serum from control and treated animals was profiled using a multiplex flow cytometry approach with antibody-coated microspheres to ascertain the abundance of inflammatory and fibrogenic proteins. Most notably, levels of effector cytokines contributing to fibrosis or a type 2 helper T cell (Th2) milieu (i.e., Interleukins IL-5, IL-10, IL-17) were more abundant in $M y d 88^{-/-}$animals with late lung injury than in similarly aged and treated WT mice (Figure 6). Also, IL-6 and Macrophage inflammatory protein 1-alpha/ C-C motif chemokine 3 (MIP1a/CCL3) levels in irradiated $M y d 88^{-1-}$ mice were significantly diminished in comparison to WT mice at 18 and 13 wpi, respectively. At 18 wpi, in WT mice, IL-1a, IL-1 $\beta$ and MIP1 $a$ levels were lowest, while these factors did not drop in $M y d 88^{-1-}$ mice. There were no significant differences over time or between genotypes in the levels of MCP1/CCL2, basic Fibroblast growth factor (bFGF), Vascular endothelial growth factor (VEGF), or IL-2, although a trend of increased IL-2 in $M y d 88^{-1-}$ mice from 13-24 wpi was apparent. Radiation-induced chronic lung injury in Myd88deficient animals appeared to correlate with dysregulated production of immune factors.

\section{Discussion}

The critical need to protect host tissue from secondary consequences of irradiation as well as the current climate of proliferating bioterrorism dangers, whether from irradiation alone or in combination with pathogenic or other damaging agents, demands the investigation and application of host defense tools to address how threats may be alleviated and host survival promoted (DiCarlo et al. 2008). Earlier studies demonstrated that TLR signaling induced by flagellin plays a protective role for host survival when subjects are treated with irradiation (Burdelya et al. 2008, Sanders et al. 2008, Vijay-Kumar et al. 2008, Janot et al. 2009). Notably, TLR signaling has been shown to be induced by a variety of endogenous stimuli, including heat shock proteins, high mobility group protein B1 (HMGB1), nucleic acids, and hyaluronan (Jiang et al. 2006), in addition to exogenous agonists or pathogens. There is strong evidence that MyD88 is a key control element for innate immune signaling in response to pathogenic stimuli (Skerrett et al. 2004, Rudd et al. 2007, Bretz et al. 2008, Rahman et al. 2008) as well as mechanical and environmental stresses (Togbe et al. 2006, Williams et al. 2007, Babcock et al. 2008, Doz et al. 2008). Due to its critical position as 
signaling mediator for host immune responses initiated by TLR, IL-1R, or IL-18R signaling pathways, MyD88 provides a common fulcrum to examine host defense responses.

Based on this body of work, it was reasonable to expect that MyD88 would play a significant role in regulating inflammation in the lung, but its role in the repair or exacerbation of lung injury caused by irradiation exposure had not been previously described. Therefore, we aimed to broaden the understanding of TLR signaling mediators and application to the realms of cancer radiotherapy and nuclear bioterrorist threats. In this study we undertook an investigation of MyD88 in both acute and long-term lung injury since the lung is exquisitely sensitive to the damaging effects of irradiation (Milano et al. 2007). We found that MyD88 is important for host survival from radiation-induced injury and that in the absence of MyD88, cells accumulated in the lung, which ultimately displayed a fibrotic phenotype by 24 weeks following radiation exposure. These results demonstrate that MyD88 is important for regulating non-infectious inflammatory processes so as to promote healthy tissue regeneration in the lung after irradiation. In addition to the wellknown role of MyD88 in regulating immune responses to direct and sudden injury, there is evidence suggesting its role in regulating hematopoeisis and cell replenishment programs (Nagai et al. 2006, McGettrick and O' Neill 2007). Since we found some differences in cell composition, airway physiology and cytokines at basal state in WT and Myd88-null mice, further study is required to assess whether the phenotypes are due directly to MyD88 deficiency in the airway or as a consequence of altering the initial basal environment.

These results are consistent with previous reports of injury caused by irradiation of C57BL/6 animals, a strain commonly known to be susceptible to fibrosis, but not to pneumonitis (Dileto and Travis 1996, Williams et al. 2010). The median survival time of $M y d 88^{-/-}$mice of 27 wpi occurred just before the 28-30 week mark that has signaled significant respiratory distress and change in breathing rates and survival in C57BL/6 mice receiving 12-20 Gy $\gamma$ irradiation (Liao et al. 1995, Jackson et al. 2010). For this study, pathology found in lungs of irradiated C57BL/6 mice was patchy and variable, unlike other reports detailing fibrotic loci appearing and accumulating between 16-24 weeks post exposure (Chiang et al. 2005) with the mice recovering with minimal defects. In contrast, collagen-filled foci were localized to the sub-pleura in irradiated $M y d 88^{--}$animals at 18 weeks and increased in density by 27 weeks. Also, proinflammatory factors such as IL-6, CXCL1/KC, and granulocyte colonystimulating factor G-CSF have been shown to be hallmarks of inflammation in irradiated C57BL/6 mice, with a strong correlation between the abundance of each factor in lung and serum (coefficient of determination, $R^{2}$, between 0.81 and 0.98) (Ao et al. 2009). In the absence of MyD88, transcription regulation of $116, C c 12 / \mathrm{Mcp} 1$ and $C x c 11 / K C$ was diminished during the initial times post injury and the abundance of these NF- $\kappa B$-induced factors was dysregulated during the chronic injury phase. Although other differences in immediate acute stress responses were not addressed (i.e., thermoregulation and oxygenation), it seems unlikely that these would predominate over the genetic difference (i.e., absence of MyD88) that contributes to the chronic inflammatory phenotype found in Myd $88^{-/-}$animals.

Although we were surprised initially to see persisting cellular infiltrate and collagen deposition in the $\mathrm{Myd} 88^{-/-}$survivors of radiation lung injury in the context of diminished abundance of proinflammatory mRNA (i.e., II6, Ccl2/Mcp1), others have reported observations of exacerbated lung injury and inflammatory cell accumulation in $M y d 88^{-1-}$ mice due to infection with pulmonary-specific pathogens, Pseudomonas aeruginosa (Skerrett et al. 2004) or Aspergillus fumigatus (Bretz et al. 2008). Other investigators have described exacerbated lung injury in $M y d 88^{-1-}$ animals using the noninfectious treatment of chemotherapeutic bleomycin (BLM). Intratracheal instillation of BLM, an agent that damages tissues through oxidant-mediated DNA attack, has been used primarily in animal 
studies (Chua et al. 2005) as a model of idiopathic pulmonary fibrosis. Lung sections from BLM-treated $\mathrm{Myd} 88^{-/-}$mice revealed enhanced injury consisting of accumulation of inflammatory cells, thickening of interstitial spaces, an increase in total protein in bronchoalveolar lavage fluid (BALF) and elevated numbers of apoptotic cells (Jiang et al. 2005, Gasse et al. 2007). Likewise, BLM treatment of Tnf-deficient mice resulted in greater histopathology in the lungs and accumulation of inflammatory cells with no reduction of collagen deposition from wild type controls (Kuroki et al. 2003). This is significant as TNF production is dependent on NF- $\mathrm{KB}$ activation which is regulated by MyD88-mediated signaling. Our results support the conclusion that MyD88 activity is important for regulating inflammation that stimulates tissue repair, thereby protecting the lung against progressive injury.

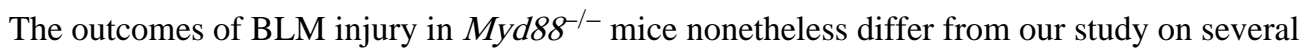
fronts. With irradiation, we found evidence of fibrosis at 24-27 wpi with collagen deposition

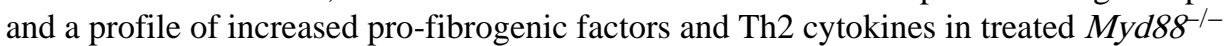
mice, while BLM treatment of $M y d 88^{-/-}$resulted in less fibrosis and reduced cellular infiltrate in comparison to treated WT mice (Gasse et al. 2007). In both studies, production of proinflammatory factors (IL-6, CXCL1/KC, MPO) was reduced. We suggest that the development of fibrosis in the Myd88-null mice surviving for 24-27 wpi is due at least in part from the persisting cellular infiltrate and changes in extracellular matrix perhaps attributed to activation of the epithelium (Rube et al. 2005, Yang et al. 2007). In fact, the abundance of Th2 and regulatory proteins (IL-5, IL-10, IL-17, IL-2) in serum of $M y d 88^{-/-}$ mice at 13-27 wpi promotes the development of a fibrogenic program (Westermann et al. 1999), marked by the upregulation of Has 2 and Has3 (Li et al. 2000, Bai et al. 2005). These results are consistent with other studies reporting the biologic correlation of the induction of Th2 cytokines and chemokines with the development of fibrosis (Belperio et al. 2004, Ma et al. 2006, Chapoval et al. 2007, Yoshizaki et al. 2010).

This initial whole organism study underscores the important benefits of MyD88 to tissue recovery from radiation-induced pulmonary injury. We have shown that mice lacking MyD88 are susceptible to radiation-induced lung injury and fibrosis, mediated through dysregulated immune responses. Additional studies not detailed here reveal that irradiation of animals defective in IL-1 $\beta$ regulation (i.e., Pyrin and caspase recruitment domaincontaining or Pycard-null mice) does not result in a chronic fibrotic phenotype, suggesting that inflammation regulatory pathways contribute differentially to injury repair and/or maintenance of immune responsiveness to radiation. Further studies should target the mechanism of protection afforded by MyD88 in order to propel research toward the development of therapeutics and mitigating agents against the detrimental effects of radiation.

\section{Acknowledgments}

The authors thank Dr Stavros Garantziotis, (NIEHS, Research Triangle Park, NC) for helpful discussions; Stephen D. Hoyle, Michael E. Kight and Jeffrey C. Hale for technical assistance in this project; Steven Budd for morphometry assessments and Richard J. Norris for expertise in operating the FlexiVent system. This research project is supported by the Radiation Countermeasures Center of Research Excellence (RadCCORE) and Immunobiology of Acute Environmental Asthma (U19-AI077437). The authors gratefully acknowledge grant support from NIH: JPYT (NIH U19-AI067798); SLT (NIH HL071802); WJB (NIH AI061059). Please note: A portion of the data with control WT mice has been included in a manuscript focused on the inflammasome molecule nucleotide binding domain leucine rich repeat protein family, pyrin domain containing 3 (NLRP3) and its role in protection against late pulmonary injury and fibrosis, as these studies were performed concurrently. 


\section{References}

Abid SH, Malhotra V, Perry MC. Radiation-induced and chemotherapy- induced pulmonary injury. Current Opinion in Oncology. 2001; 13:242-248. [PubMed: 11429481]

Akazawa T, Masuda H, Saeki Y, Matsumoto M, Takeda K, Tsujimura K, Kuzushima K, Takahashi T, Azuma I, Akira S, et al. Adjuvant-mediated tumor regression and tumor-specific cytotoxic response are impaired in MyD88-deficient mice. Cancer Research. 2004; 64:757-764. [PubMed: 14744795]

Akira S, Takeda K. Functions of toll-like receptors: Lessons from KO mice. Comptes Rendus Biologies. 2004; 327:581-589. [PubMed: 15330257]

Ao X, Zhao L, Davis MA, Lubman DM, Lawrence TS, Kong FM. Radiation produces differential changes in cytokine profiles in radiation lung fibrosis sensitive and resistant mice. Journal of Hematology and Oncology. 2009; 2:6-17. [PubMed: 19187543]

Arnett HA, Hellendall RP, Matsushima GK, Suzuki K, Laubach VE, Sherman P, Ting JP. The protective role of nitric oxide in a neurotoxicant-induced demyelinating model. Journal of Immunology. 2002; 168:427-433.

Babcock AA, Toft-Hansen H, Owens T. Signaling through MyD88 regulates leukocyte recruitment after brain injury. Journal of Immunology. 2008; 181:6481-6490.

Bai KJ, Spicer AP, Mascarenhas MM, Yu L, Ochoa CD, Garg HG, Quinn DA. The role of hyaluronan synthase 3 in ventilator-induced lung injury. American Journal of Respiratory and Critical Care Medicine. 2005; 172:92-98. [PubMed: 15790861]

Belperio JA, Dy M, Murray L, Burdick MD, Xue YY, Strieter RM, Keane MP. The role of the Th2 CC chemokine ligand CCL17 in pulmonary fibrosis. Journal of Immunology. 2004; 173:4692-4698.

Beutler B. Inferences, questions and possibilities in Toll-like receptor signalling. Nature. 2004; 430:257-263. [PubMed: 15241424]

Bjorkbacka H, Kunjathoor VV, Moore KJ, Koehn S, Ordija CM, Lee MA, Means T, Halmen K, Luster $\mathrm{AD}$, Golenbock DT, et al. Reduced atherosclerosis in MyD88-null mice links elevated serum cholesterol levels to activation of innate immunity signaling pathways. Nature Medicine. 2004; 10:416-421.

Bretz C, Gersuk G, Knoblaugh S, Chaudhary N, Randolph-Habecker J, Hackman RC, Staab J, Marr KA. MyD88 signaling contributes to early pulmonary responses to Aspergillus fumigatus. Infection and Immunity. 2008; 76:952-958. [PubMed: 18039832]

Burdelya LG, Krivokrysenko VI, Tallant TC, Strom E, Gleiberman AS, Gupta D, Kurnasov OV, Fort FL, Osterman AL, Didonato JA, et al. An agonist of toll-like receptor 5 has radioprotective activity in mouse and primate models. Science. 2008; 320:226-230. [PubMed: 18403709]

Cao Z, Henzel WJ, Gao X. IRAK: A kinase associated with the interleukin-1 receptor. Science. 1996; 271:1128-1131. [PubMed: 8599092]

Chapoval SP, Al-Garawi A, Lora JM, Strickland I, Ma B, Lee PJ, Homer RJ, Ghosh S, Coyle AJ, Elias JA. Inhibition of NF-kappaB activation reduces the tissue effects of transgenic IL-13. Journal of Immunology. 2007; 179:7030-7041.

Chen Y, Williams J, Ding I, Hernady E, Liu W, Smudzin T, Finkelstein JN, Rubin P, Okunieff P. Radiation pneumonitis and early circulatory cytokine markers. Seminars in Radiation Oncology. 2002; 12(1 Suppl 1):26-33. [PubMed: 11917281]

Chiang CS, Liu WC, Jung SM, Chen FH, Wu CR, McBride WH, Lee CC, Hong JH. Compartmental responses after thoracic irradiation of mice: Strain differences. International Journal of Radiation Oncology *Biology *Physics. 2005; 62:862-871.

Chua F, Gauldie J, Laurent GJ. Pulmonary fibrosis: Searching for model answers. American Journal of Respiratory Cell and Molecular Biology. 2005; 33:9-13. [PubMed: 15964990]

DiCarlo AL, Hatchett RJ, Kaminski JM, Ledney GD, Pellmar TC, Okunieff P, Ramakrishnan N. Medical countermeasures for radiation combined injury: Radiation with burn, blast, trauma and/or sepsis. Report of an NIAID Workshop, March 26-27, 2007. Radiation Research. 2008; 169:712721. [PubMed: 18494548]

Dileto CL, Travis EL. Fibroblast radiosensitivity in vitro and lung fibrosis in vivo: Comparison between a fibrosis-prone and fibrosis-resistant mouse strain. Radiation Research. 1996; 146:6167. [PubMed: 8677299] 
Doz E, Noulin N, Boichot E, Guenon I, Fick L, Le Bert M, Lagente V, Ryffel B, Schnyder B, Quesniaux VF, et al. Cigarette smoke-induced pulmonary inflammation is TLR4/MyD88 and IL-1R1/MyD88 signaling dependent. Journal of Immunology. 2008; 180:1169-1178.

Gasse P, Mary C, Guenon I, Noulin N, Charron S, Schnyder-Candrian S, Schnyder B, Akira S, Quesniaux VF, Lagente V, et al. IL-1R1/MyD88 signaling and the inflammasome are essential in pulmonary inflammation and fibrosis in mice. Journal of Clinical Investigation. 2007; 117:37863799. [PubMed: 17992263]

Glaab T, Taube C, Braun A, Mitzner W. Invasive and noninvasive methods for studying pulmonary function in mice. Respiratory Research. 2007; 8:63- 72. [PubMed: 17868442]

Goldstein DR, Tesar BM, Akira S, Lakkis FG. Critical role of the Toll-like receptor signal adaptor protein MyD88 in acute allograft rejection. Journal of Clinical Investigation. 2003; 111:15711578. [PubMed: 12750407]

Graves PR, Siddiqui F, Anscher MS, Movsas B. Radiation pulmonary toxicity: From mechanisms to management. Seminars in Radiation Oncology. 2010; 20:201-207. [PubMed: 20685583]

Hacker H, Vabulas RM, Takeuchi O, Hoshino K, Akira S, Wagner H. Immune cell activation by bacterial CpG-DNA through myeloid differentiation marker 88 and tumor necrosis factor receptorassociated factor (TRAF)6. Journal of Experimental Medicine. 2000; 192:595-600. [PubMed: 10952730]

Hill RP. Radiation effects on the respiratory system. The British Journal of Radiology Supplementals/ Special Issues. 2005; 27:75-81.

Irvin CG, Bates JH. Measuring the lung function in the mouse: The challenge of size. Respiratory Research. 2003; 4:4-12. [PubMed: 12783622]

Jackson IL, Vujaskovic Z, Down JD. Revisiting strain-related differences in radiation sensitivity of the mouse lung: Recognizing and avoiding the confounding effects of pleural effusions. Radiation Research. 2010; 173:10- 20. [PubMed: 20041755]

Janot L, Sirard JC, Secher T, Noulin N, Fick L, Akira S, Uematsu S, Didierlaurent A, Hussell T, Ryffel B, et al. Radioresistant cells expressing TLR5 control the respiratory epithelium's innate immune responses to flagellin. European Journal of Immunology. 2009; 39:1587-1596. [PubMed: 19424969]

Jha S, Srivastava SY, Brickey WJ, Iocca H, Toews A, Morrison JP, Chen VS, Gris D, Matsushima GK, Ting JP. The inflammasome sensor, NLRP3, regulates CNS inflammation and demyelination via caspase-1 and interleukin-18. The Journal of Neuroscience. 2010; 30:15811-15820. [PubMed: 21106820]

Jiang D, Liang J, Li Y, Noble PW. The role of Toll-like receptors in non-infectious lung injury. Cell Research. 2006; 16:693-701. [PubMed: 16894359]

Jiang D, Liang J, Fan J, Yu S, Chen S, Luo Y, Prestwich GD, Mascarenhas MM, Garg HG, Quinn DA, et al. Regulation of lung injury and repair by Toll-like receptors and hyaluronan. Nature Medicine. 2005; 11:1173-1179.

Junqueira LC, Bignolas G, Brentani RR. Picrosirius staining plus polarization microscopy, a specific method for collagen detection in tissue sections. Histochemical Journal. 1979; 11:447-455. [PubMed: 91593]

Keane MP, Belperio JA, Moore TA, Moore BB, Arenberg DA, Smith RE, Burdick MD, Kunkel SL, Strieter RM. Neutralization of the CXC chemokine, macrophage inflammatory protein-2, attenuates bleomycin-induced pulmonary fibrosis. Journal of Immunology. 1999; 162:5511-5518.

Kenny EF, O' Neill LA. Signalling adaptors used by Toll-like receptors: An update. Cytokine. 2008; 43:342-349. [PubMed: 18706831]

Kuroki M, Noguchi Y, Shimono M, Tomono K, Tashiro T, Obata Y, Nakayama E, Kohno S. Repression of bleomycin-induced pneumopathy by TNF. Journal of Immunology. 2003; 170:567574.

Li L. Regulation of innate immunity signaling and its connection with human diseases. Current Drug Targets-Inflammation and Allergy. 2004; 3:81-86. [PubMed: 15032644]

Li Y, Rahmanian M, Widstrom C, Lepperdinger G, Frost GI, Heldin P. Irradiation-induced expression of hyaluronan (HA) synthase 2 and hyaluronidase 2 genes in rat lung tissue accompanies active 
turnover of HA and induction of types I and III collagen gene expression. American Journal of Respiratory Cell and Molecular Biology. 2000; 23:411-418. [PubMed: 10970834]

Liao ZX, Travis EL, Tucker SL. Damage and morbidity from pneumonitis after irradiation of partial volumes of mouse lung. International Journal of Radiation Oncology *Biology *Physics. 1995; 32:1359-1370.

Liebermann DA, Hoffman B. Myeloid differentiation (MyD) primary response genes in hematopoiesis. Blood Cells, Molecules and Diseases. 2003; 31:213-228.

Lovgren AK, Jania LA, Hartney JM, Parsons KK, Audoly LP, Fitzgerald GA, Tilley SL, Koller BH. COX-2-derived prostacyclin protects against bleomycin-induced pulmonary fibrosis. American Journal of Physiology-Lung Cellular and Molecular Physiology. 2006; 291:L144-156. [PubMed: 16473862]

Ma B, Blackburn MR, Lee CG, Homer RJ, Liu W, Flavell RA, Boyden L, Lifton RP, Sun CX, Young $\mathrm{HW}$, et al. Adenosine metabolism and murine strain-specific IL-4-induced inflammation, emphysema, and fibrosis. Journal of Clinical Investigation. 2006; 116:1274-1283. [PubMed: 16670768]

McBride WH, Vegesna V. The role of T-cells in radiation pneumonitis after bone marrow transplantation. International Journal of Radiation Biology. 2000; 76:517-521. [PubMed: 10815632]

McGettrick AF, O' Neill LA. Toll-like receptors: Key activators of leucocytes and regulator of haematopoiesis. British Journal of Haematology. 2007; 139:185-193. [PubMed: 17897294]

Medzhitov R, Janeway C Jr. The Toll receptor family and microbial recognition. Trends in Microbiology. 2000; 8:452-456. [PubMed: 11044679]

Milano MT, Constine LS, Okunieff P. Normal tissue tolerance dose metrics for radiation therapy of major organs. Seminars in Radiation Oncology. 2007; 17:131-140. [PubMed: 17395043]

Miura G, Awaya H, Matsumoto T, Tanaka N, Matsunaga N. Does granulocyte colony-stimulating factor exacerbate radiation-induced acute lung injury in rats? Radiation Medicine. 2000; 18:227232. [PubMed: 11246997]

Nagai Y, Garrett KP, Ohta S, Bahrun U, Kouro T, Akira S, Takatsu K, Kincade PW. Toll-like receptors on hematopoietic progenitor cells stimulate innate immune system replenishment. Immunity. 2006; 24:801-812. [PubMed: 16782035]

Nakayama Y, Makino S, Fukuda Y, Min KY, Shimizu A, Ohsawa N. Activation of lavage lymphocytes in lung injuries caused by radiotherapy for lung cancer. International Journal of Radiation Oncology *Biology *Physics. 1996; 34:459-467.

Rahman AH, Cui W, Larosa DF, Taylor DK, Zhang J, Goldstein DR, Wherry EJ, Kaech SM, Turka LA. MyD88 plays a critical T cell-intrinsic role in supporting CD8 T cell expansion during acute lymphocytic choriomeningitis virus infection. Journal of Immunology. 2008; 181:3804-3810.

Roberts CM, Foulcher E, Zaunders JJ, Bryant DH, Freund J, Cairns D, Penny R, Morgan GW, Breit SN. Radiation pneumonitis: A possible lymphocyte-mediated hypersensitivity reaction. Annals of Internal Medicine. 1993; 118:696-700. [PubMed: 8460855]

Roses RE, Xu M, Koski GK, Czerniecki BJ. Radiation therapy and Toll-like receptor signaling: Implications for the treatment of cancer. Oncogene. 2008; 27:200-207. [PubMed: 18176601]

Rube CE, Wilfert F, Uthe D, Schmid KW, Knoop R, Willich N, Schuck A, Rube C. Modulation of radiation-induced tumour necrosis factor alpha (TNF-alpha) expression in the lung tissue by pentoxifylline. Radiotherapy and Oncology. 2002; 64:177-187. [PubMed: 12242128]

Rube CE, Uthe D, Wilfert F, Ludwig D, Yang K, Konig J, Palm J, Schuck A, Willich N, Remberger $\mathrm{K}$, et al. The bronchiolar epithelium as a prominent source of pro-inflammatory cytokines after lung irradiation. International Journal of Radiation Oncology *Biology *Physics. 2005; 61:14821492.

Rubin P, Johnston CJ, Williams JP, McDonald S, Finkelstein JN. A perpetual cascade of cytokines postirradiation leads to pulmonary fibrosis. International Journal of Radiation Oncology *Biology *Physics. 1995; 33:99-109.

Rudd BD, Schaller MA, Smit JJ, Kunkel SL, Neupane R, Kelley L, Berlin AA, Lukacs NW. MyD88mediated instructive signals in dendritic cells regulate pulmonary immune responses during respiratory virus infection. Journal of Immunology. 2007; 178:5820-5827. 
Sanders CJ, Moore DA 3rd, Williams IR, Gewirtz AT. Both radioresistant and hemopoietic cells promote innate and adaptive immune responses to flagellin. Journal of Immunology. 2008; 180:7184-7192.

Sharplin J, Franko AJ. A quantitative histological study of strain-dependent differences in the effects of irradiation on mouse lung during the intermediate and late phases. Radiation Research. 1989; 119:15-31. [PubMed: 2756106]

Skerrett SJ, Liggitt HD, Hajjar AM, Wilson CB. Cutting edge: Myeloid differentiation factor 88 is essential for pulmonary host defense against Pseudomonas aeruginosa but not Staphylococcus aureus. Journal of Immunology. 2004; 172:3377-3381.

Smith RE, Strieter RM, Phan SH, Lukacs NW, Huffnagle GB, Wilke CA, Burdick MD, Lincoln P, Evanoff H, Kunkel SL. Production and function of murine macrophage inflammatory protein-1 alpha in bleomycin-induced lung injury. Journal of Immunology. 1994; 153:4704-4712.

Togbe D, Aurore G, Noulin N, Quesniaux VF, Schnyder-Candrian S, Schnyder B, Vasseur V, Akira S, Hoebe K, Beutler B, et al. Nonredundant roles of TIRAP and MyD88 in airway response to endotoxin, independent of TRIF, IL-1 and IL-18 pathways. Laboratory Investigation. 2006; 86:1126- 1135. [PubMed: 16983331]

Vijay-Kumar M, Aitken JD, Sanders CJ, Frias A, Sloane VM, Xu J, Neish AS, Rojas M, Gewirtz AT. Flagellin treatment protects against chemicals, bacteria, viruses, and radiation. Journal of Immunology. 2008; 180:8280-8285.

Wang L, Guo Y, Huang WJ, Ke X, Poyet JL, Manji GA, Merriam S, Glucksmann MA, DiStefano PS, Alnemri ES, et al. Card10 is a novel caspase recruitment domain/membrane-associated guanylate kinase family member that interacts with BCL10 and activates NF-kappa B. Journal of Biological Chemistry. 2001; 276:21405-21409. [PubMed: 11259443]

Westermann W, Schobl R, Rieber EP, Frank KH. Th2 cells as effectors in postirradiation pulmonary damage preceding fibrosis in the rat. International Journal of Radiation Biology. 1999; 75:629638. [PubMed: 10374945]

Williams AS, Leung SY, Nath P, Khorasani NM, Bhavsar P, Issa R, Mitchell JA, Adcock IM, Chung KF. Role of TLR2, TLR4, and MyD88 in murine ozone-induced airway hyperresponsiveness and neutrophilia. Journal of Applied Physiology. 2007; 103:1189-1195. [PubMed: 17626835]

Williams JP, Brown SL, Georges GE, Hauer-Jensen M, Hill RP, Huser AK, Kirsch DG, Macvittie TJ, Mason KA, Medhora MM, et al. Animal models for medical countermeasures to radiation exposure. Radiation Research. 2010; 173:557- 578. [PubMed: 20334528]

Xu Y, Tao X, Shen B, Horng T, Medzhitov R, Manley JL, Tong L. Structural basis for signal transduction by the Toll/interleukin-1 receptor domains. Nature. 2000; 408:111-115. [PubMed: 11081518]

Yang K, Palm J, Konig J, Seeland U, Rosenkranz S, Feiden W, Rube C, Rube CE. Matrix-MetalloProteinases and their tissue inhibitors in radiation-induced lung injury. International Journal of Radiation Biology. 2007; 83:665-676. [PubMed: 17729161]

Yoshizaki A, Yanaba K, Iwata Y, Komura K, Ogawa A, Akiyama Y, Muroi E, Hara T, Ogawa F, Takenaka M, et al. Cell adhesion molecules regulate fibrotic process via Th1/Th2/Th17 cell balance in a bleomycin-induced scleroderma model. Journal of Immunology. 2010; 185:25022515. 
A

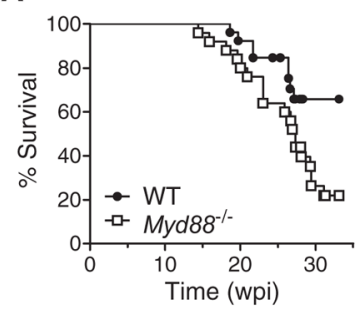

C
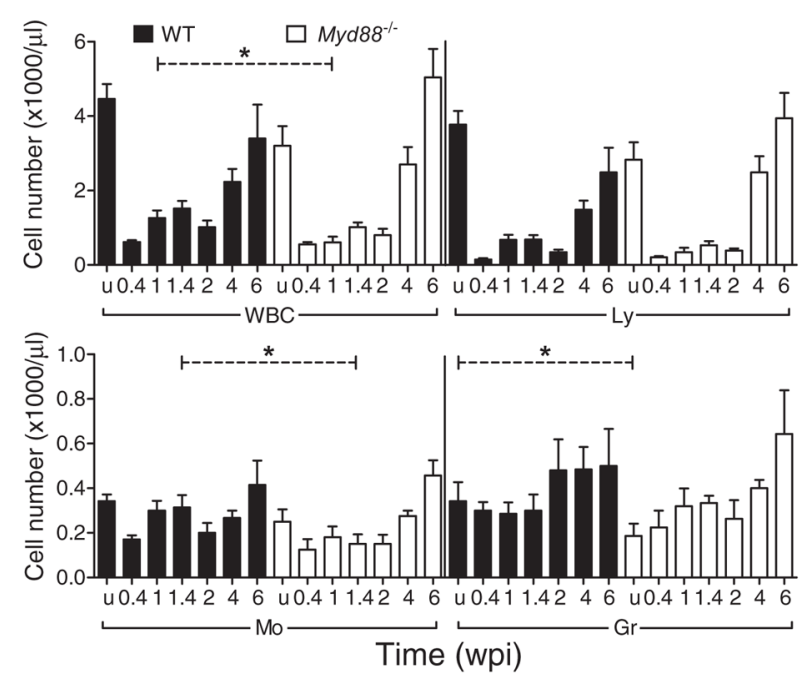

Figure 1.

MyD88 provides protection against mortality linked to exposure to thoracic targeted $\gamma$ irradiation. (A) Loss of MyD88 produces a strain susceptible to radiation-induced lung injury. A Kaplan-Meier survival analysis of irradiated $\mathrm{Myd}^{-1-}(n=25)$ or WT mice $(n=$ 26 ) is shown. The median survival time (MST) for $M y d 88^{-/-}$is 27.3 weeks post irradiation (wpi). Comparison of the survival curves using the log rank test (Mantel-Cox) reveals a significant difference $(p<0.05)$, with a hazard ratio of 2.237. Peripheral blood cell composition of white blood cells (WBC), lymphocytes (Ly), monocytes (Mo) and granulocytes $(\mathrm{Gr})$ in mice at baseline (B) and after radiation (C) is similar between WT and $M y d 88^{-/-}$mice. Cells in peripheral blood samples were quantified using Heska's Animal Blood Counter. The average absolute numbers (in 1000s) are plotted with significant differences between WT (solid) and $M y d 88^{-/-}$(open) at indicated times shown by dotted line with error bars indicating SEM for $n=7$ per time for each genotype in (C) $(* P<0.05$; $\mathrm{u}$, untreated). 

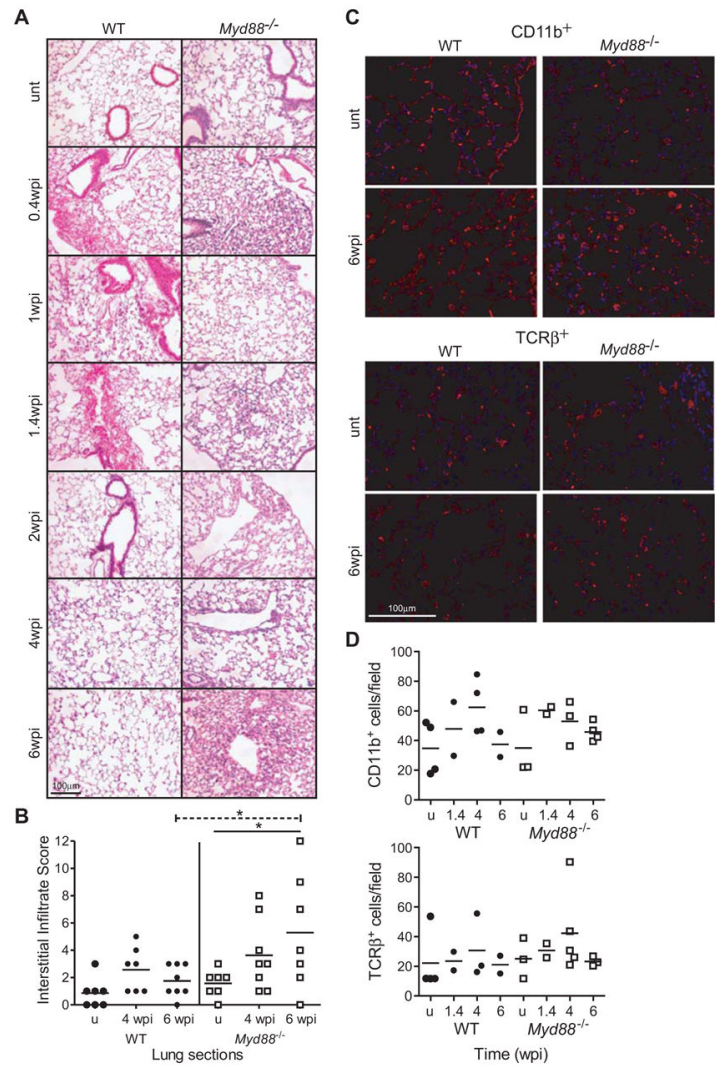

Figure 2.

Cellularity in lungs of irradiated mice is enhanced during acute injury. (A) Representative images of H\&E stained lung sections of WT (left) and $M y d 88^{-/-}$(right) mice, untreated (unt) and weeks post irradiation (wpi). (Original magnification 200×) (B) The interstitial infiltrate of alveolar spaces in unirradiated (u) WT (circle) and $M y d 88^{-/-}$(square) mice and at 4-6 wpi was scored by reviewers unaware of tissue identity. Individual samples are represented by each symbol with the averaged cumulative score represented by the bar. ${ }^{*} P<$ 0.05 indicates significantly different means between WT and $M y d 88^{-/-}$at 6 wpi (dotted line) and for $M y d 88^{-/-}$over time (solid line). (C) Immunohistochemical analysis of lungs revealed elevated numbers of T lymphocytic $\left(\mathrm{TCR} \beta^{+}\right)$and myeloid $\left(\mathrm{CD} 11 \mathrm{~b}^{+}\right)$cells in lungs of irradiated animals at 6 weeks. Representative images are shown. (Original magnification 200×, scale bar 100 micron) (D) Numbers of antibody-positive DAPI-stained cells were counted in 5-7 fields for each biological sample, with average CD $11 b^{+}$or $\mathrm{TCR}^{+}$cells/field per sample indicated by symbol and mean for each time plotted for untreated (u) samples and from 1.4-6 wpi. 
A
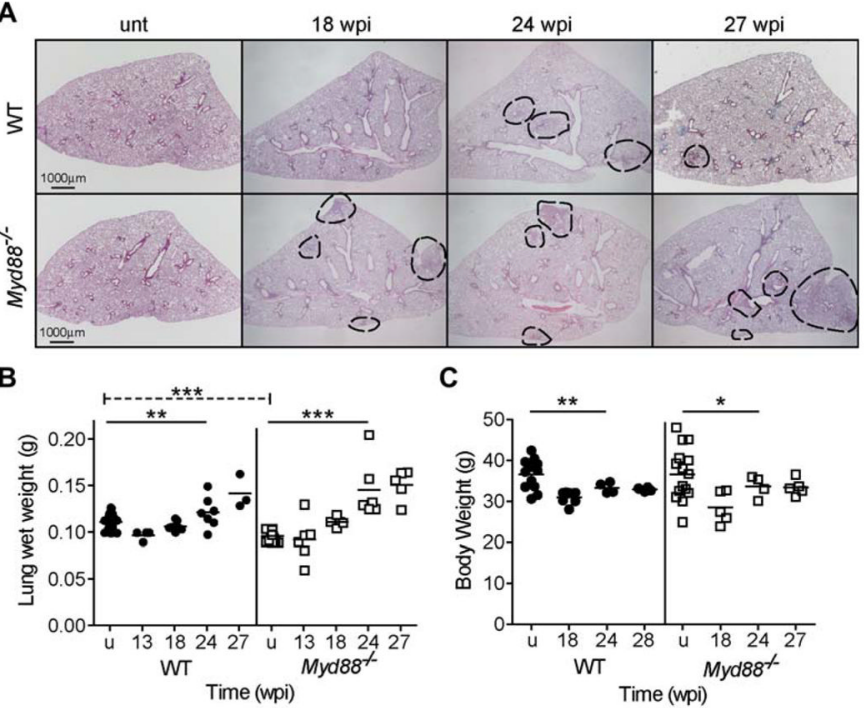

C
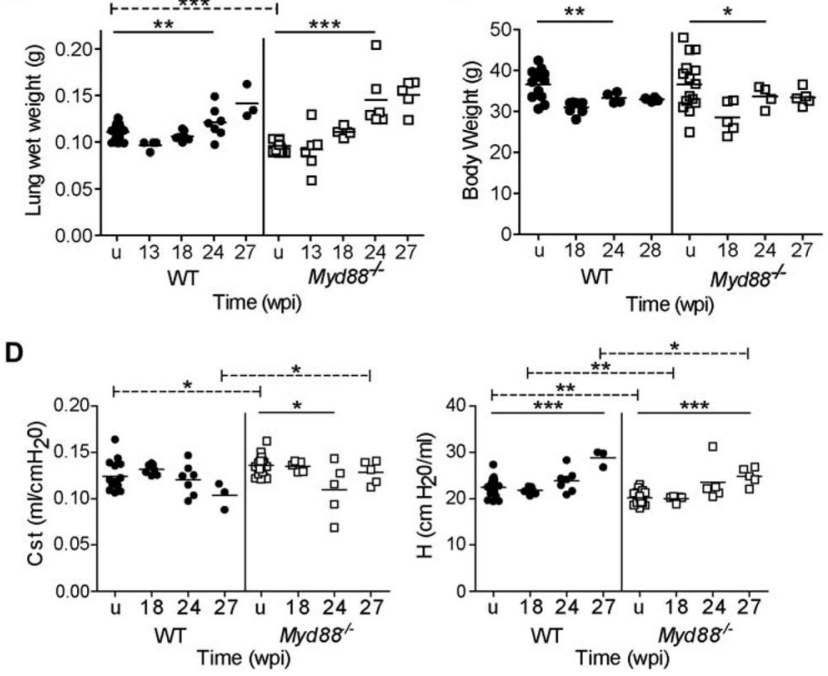

Figure 3.

Late lung injury persists in irradiated $\mathrm{Myd} 8 \mathrm{8}^{-/-}$animals. (A) Fixed lung sections were stained with $H \& E$ and representative images are shown for untreated (unt) and samples from mice at 18-27 wpi. (Original magnification 40x). Foci of parenchymal damage are marked with dotted lines. Changes in lung wet weight (B) and body weight (C) were monitored. (D) Irradiated $\mathrm{Myd} 88^{-/-}$mice display impaired airway mechanics. The static compliance (Cst) and tissue elastance $(\mathrm{H})$ properties were determined through pressure-volume loops and forced oscillatory mechanics. Each data point in B-D represents an individual animal (WT, solid circle; $M y d 88^{-1-}$, open square). The mean for each parameter is represented by a bar, with significant differences over time indicated by the solid bar and between genotypes at designated times by the dotted bar $(* P<0.05 ; * * P<0.005 ; * * * P<0.0005)$. 

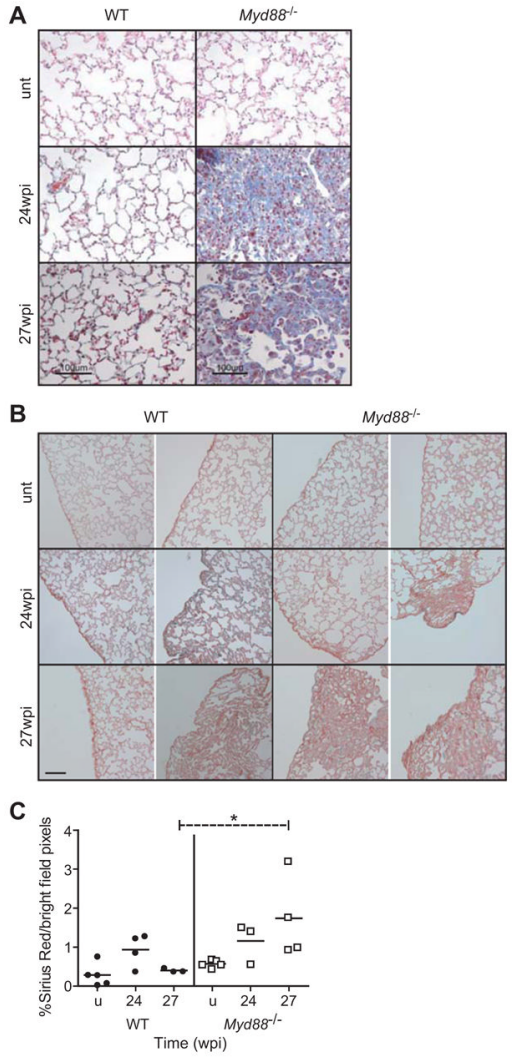

Figure 4.

Lungs in $M y d 88^{-/-}$mice display fibrotic foci of collagen deposition. Fixed lung sections were stained with H\&E (unt), Masson's Trichrome (A) or Sirius Red (B) and representative images are shown for long term WT and $M y d 88^{-1-}$ radiation survivors (24 and 27 wpi)

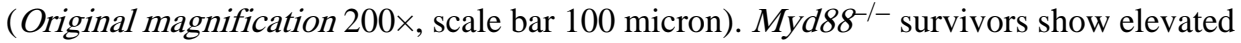
levels of Sirius Red polarized signal (C) at 27 wpi over baseline and WT at similar times. The assessment of Sirius red signal was executed by reviewers unaware of the identity of the tissue section. Each data point represents an individual animal, with the mean indicated by a bar and significant differences between genotypes at designated times by the dotted bar $(* P$ $<0.05)$. 


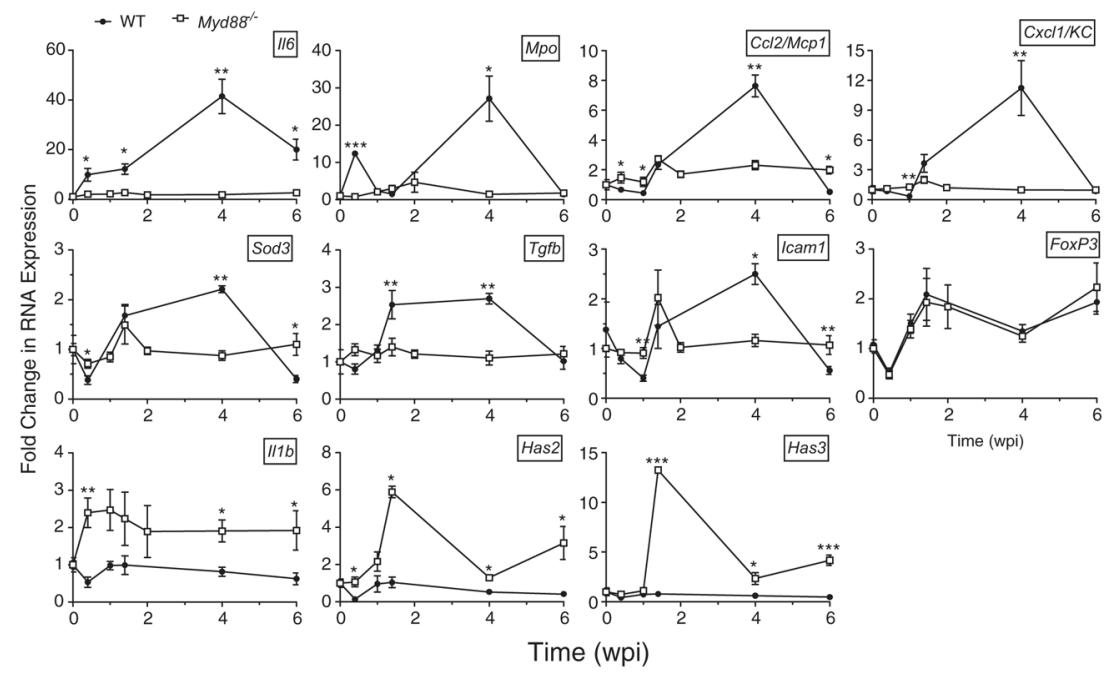

Figure 5.

Regulation of RNA expression of proinflammatory cytokines and chemokines is altered in irradiated $\mathrm{Myd} 88^{-/-}$animals during acute injury. Transcripts encoding proinflammatory cytokines $(I 16, I 11 b)$, chemokines $(C c 12 / M c p 1, C X c 11 / K C)$ as well as cell activation factors (Mpo, Sod3, Tgfb, Icam1, Foxp3, Has2, Has3) were measured using gene-specific primers and quantitative PCR approaches. Mean fold change over untreated samples of transcript levels normalized to $18 \mathrm{~S}$ rRNA are shown with error bars indicating SEM for $n=3-6$ for each group. Changes in transcript levels in $M y d 88^{-/-}$lung samples (open square) that are significantly different from WT samples (solid circle) are indicated $(* P<0.05 ; * * P<0.005$; *** $P<0.0005)$. 


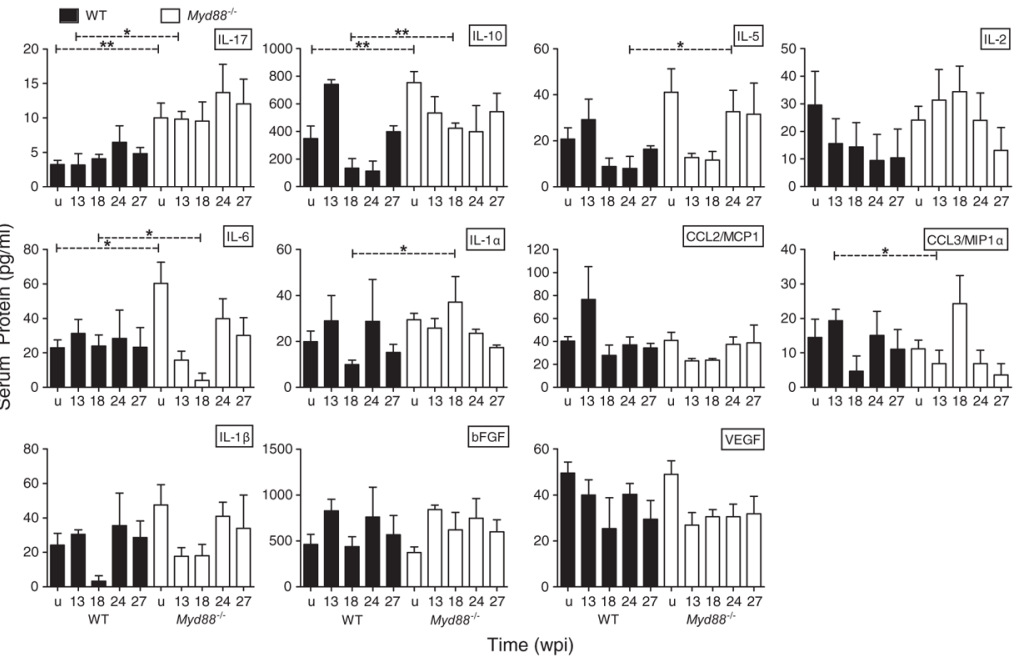

Figure 6.

Fibrosis-inducing and inflammation-suppressing cytokine levels in irradiated $M y d 88^{-1-}$ mice are elevated in serum. Proteins found in serum from WT (solid) or $\mathrm{Myd} 88^{-1-}$ (open) mice either without (u) or with irradiation (13-27 wpi) were characterized using a multiplex protein immune assay. The mean protein levels $(\mathrm{pg} / \mathrm{ml})$ are reported with error bars indicating SEM for $n=3-5$ for each group, and differences between WT and $M y d 88^{-1-}$ samples are indicated by dotted lines $(* P<0.05 ; * * P<0.005)$. 